\title{
A MODIFIED FOURIER-RITZ METHOD FOR FREE VIBRATION OF RECTANGULAR PLATES WITH ELASTIC CONSTRAINS
}

\author{
Tao Wu, Zhao Bo Chen, Jian Jun Qu \\ School of Mechatronics Engineering Harbin Institute of Technology, Harbin, China \\ Corresonding author: Zhao Bo Chen, hgdxtwxcb@163.com
}

\begin{abstract}
In this paper, a modified Fourier-Ritz method is used to study free vibration of a rectangular plate with a set of simply supported opposite sides and another set of arbitrary elastic constraints. The influence of different elastic constraint stiffness values on the modal response of the rectangular plate is also analyzed. In order to avoid that the displacement function of the rectangular plate calculated by the traditional method and its derivative may be discontinuous or non-derivable at the boundary, the displacement function is expressed in the form of the sum of standard cosine series and a periodic polynomial function. Compared with the sine series expansion, the convergence of the result is enhanced. Several sets of numerical examples with different boundary conditions are given in the article, the data shows that the results calculated by this method have good accuracy and fast convergence. In addition, this paper also analyzes the boundary conditions and discusses the influence of different spring stiffness values on the setting of boundary conditions. The results can be applied to the setting of general boundary conditions and the study of vibration control of rectangular plates.
\end{abstract}

Keywords: modified Fourier-Ritz method, rectangular plate, free vibration, boundary conditions

\section{Introduction}

As we all know, the rectangular plate structure has a wide range of applications in many fields of mechanical engineering, construction engineering, vehicle engineering and other fields. Its vibration forms mainly include bending vibration, longitudinal vibration and transverse vibration. The longitudinal and transverse modes belong to in-plane vibration, because its natural frequency is usually not in the range of the main excitation frequency, it is only considered in some special engineering applications (Du et al., 2007; He et al., 2013). On the contrary, the frequency of transverse bending vibration is easily within the external excitation frequency range, so it has a high reference value for research and practical application.

In the vibration solution of a rectangular plate with at least a pair of simply supported opposite sides, the exact solution is usually expressed in the form of trigonometric and hyperbolic functions or their combination (Han Qingkai, 2016; Cao, 1989), however, some of the constants must be determined according to the boundary conditions, which are mainly affected by the aspect ratio of the rectangular plate, the stiffness of the constrained spring, Poisson's ratio, thickness and other factors. Therefore, in some solving methods, it is forced to repeatedly solve the equations to get solutions for different boundary conditions. Many researchers have adopted the Rayleigh-Ritz method to solve the natural frequency of transverse vibration of a rectangular plate with elastic constraints. Gavalas and El-Raheb (2014) and Ilanko et al. (2014) proved the accuracy of this method and adopted this method to solve high-order modes of rectangular plates under certain conditions. Wang et al. (2006) used the differential quadrature (DQ) method to calculate vibration and buckling of rectangular plates with S-C-S-C (where C represents the 
clamped edge and S represents the simply supported edge, which is the same as later) boundary conditions, and gave a numerical example to prove the accuracy and convergence of the method, but the convergence of the results was oscillatory, and more terms were needed to obtain the exact solution. Bert and Devarakonda (2003) used the Galerkin method to find the solution of rectangular plates with S-S-S-S boundary conditions, however, it was difficult to ensure the closedness of the solution under the condition of transverse shear deformation. Wei et al. (2020) used the generalized superposition method to reduce the size of the stiffness matrix, and obtained a homogeneous solution to the vibration control equation of a rectangular plate, and proved its effectiveness, accuracy and convergence through examples. Banerjee et al. (2015) and Liu and Banerjee (2016) derived the dynamic stiffness matrix of a rectangular plate by solving the biharmonic equation, so as to more accurately obtain the natural frequency and vibration mode of free vibration of the rectangular plate, and proved its accuracy by selecting modal vibration samples, but complex symbolic calculation was required. Abdulkerim et al. (2019) studied the rectangular plate with boundary constraint of F-C-F-C (where F represents free edge) through an experimental method, and proved the effectiveness of the experimental method by comparing the experimental results with the results of a finite element numerical model. Eisenberger and Deutsch (2019) proposed a method to express the displacement function as a combination of a trigonometric function and a hyperbolic function to calculate vibration of rectangular plates under various boundary conditions. This solution does not need to separate the symmetric and antisymmetric problems, but the calculation is more complicated. Zhang et al. (2020) directly used the finite integral transformation method to obtain the analytical solution of free vibration of a rectangular thin plate. The main advantage of this method was that there was no need to re-determine the deviation function, so it had a certain universality. Li et al. (2019) combined the symplectic elasticity method with the superposition method to obtain an analytical solution to vibration of a rectangular thin plate with two adjacent free edges. The advantage of this method is that it does not need to determine the form of the solution through experience, and can solve the problems that cannot be solved by the traditional method under some specific conditions. Xing and Xu (2013) used the variable separation method to calculate the exact solution of free vibration of a rectangular thin plate under the classical boundary conditions, and gave the first six natural frequencies of the orthotropic rectangular plate, but Bahrami et al. (2014) pointed out that this method was not suitable for solving non-Levy type plates. In addition, many researchers can also obtain more accurate vibration solutions of rectangular plates by using the finite difference method, finite element method and discrete element method after appropriate modification and optimization (Najarzadeh et al., 2018; Alkhayal et al., 2019).

From the above review of the research results, it can be seen that some researchers pay attention to vibration of rectangular plates under certain constraints or classical constraints, but in practical applications, they may encounter a combination of various boundary constraints. Another part of the researchers tried to develop an algorithm that includes all boundary constraints, but there are also problems such as convergence oscillation, slow convergence and the need to repeat symbolic calculations.

This paper proposes a modified Fourier-Ritz method to solve free vibration of a rectangular plate with a set of simply supported opposite sides and another set of arbitrary elastic constraints. Similar methods have been used to solve vibration of single-beam and double-beam structures (Li, 2001; Shi et al., 2015; Hao et al., 2018; Shi et al., 2016).

In this paper, the Fourier-Ritz method is modified and extended to solve vibration of rectangular plates, that is, the displacement function of the rectangular plate is expressed as the sum of standard cosine series and a periodic polynomial function. This way, the discontinuity or non-derivability of the displacement function or its derivative at the boundary can be eliminated. Moreover, it can be applied to the solution of any boundary condition only by modifying values of some parameters without frequently modifying the solution program. In this paper, the 
accuracy and convergence are verified by comparing several numerical examples under different conditions with the existing research results. The influence of different spring stiffness values on the setting of boundary conditions is discussed, and the results are helpful to the setting of general boundary conditions. In addition, calculation results of transverse vibration of rectangular plates under some boundary conditions are given.

\section{Theoretical formulations}

As shown in Fig. 1, this paper studies vibration of a rectangular plate with elastic constraints along the edges. It is constrained by simple supports (the dashed line) in the $y=0, b$ direction and elastic supports in the $x=0, a$ direction. It includes torsional springs $\left(K_{0}, K_{a}\right)$ and linear springs $\left(k_{0}, k_{a}\right)$. We can solve vibration of rectangular plates under different boundary constraints by setting the stiffness value of elastic constraints, for example, by setting the stiffness value of two linear springs to infinity and the two torsional springs to infinitesimal in order to achieve the simple support constraint. According to Han Qingkai (2016), the free vibration differential equation of a rectangular plate is

$$
D \frac{\partial^{4} w(x, y, t)}{\partial x^{4}}+2 D \frac{\partial^{4} w(x, y, t)}{\partial x^{2} \partial y^{2}}+D \frac{\partial^{4} w(x, y, t)}{\partial y^{4}}+\rho h \frac{\partial^{2} w(x, y, t)}{\partial t^{2}}=0
$$

where $w(x, y, t)$ is the displacement function, $\rho$ is density of the rectangular plate, $D$ is bending stiffness, and $h$ is thickness of the rectangular plate. For natural vibration, the above solution can be set as

$$
w(x, y, t)=W(x, y) \mathrm{e}^{\mathrm{i} \omega t}
$$

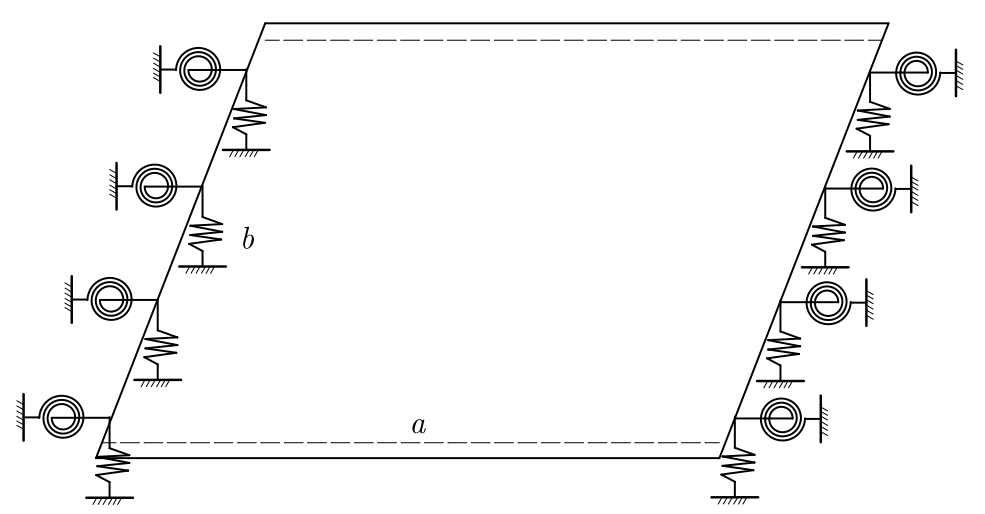

Fig. 1. Rectangular plate model with elastic constraints

By substituting (2.2) into (2.1), the following expression can be obtained

$$
D \frac{\partial^{4} W}{\partial x^{4}}+2 D \frac{\partial^{4} W}{\partial x^{2} \partial y^{2}}+D \frac{\partial^{4} W}{\partial y^{4}}-\rho h \omega^{2} W=0
$$

The boundary conditions constrained along the $x$ direction are:

- at $x=0$

$$
k_{0}=-D\left(\frac{\partial^{3} W}{\partial x^{3}}+(2-\nu) \frac{\partial^{3} W}{\partial x \partial y^{2}}\right) \quad K_{0} \frac{\partial W}{\partial x}=D\left(\frac{\partial^{2} W}{\partial x^{2}}+\nu \frac{\partial^{2} W}{\partial y^{2}}\right)
$$

- at $x=a$

$$
k_{a}=D\left(\frac{\partial^{3} W}{\partial x^{3}}+(2-\nu) \frac{\partial^{3} W}{\partial x \partial y^{2}}\right) \quad K_{a} \frac{\partial W}{\partial x}=-D\left(\frac{\partial^{2} W}{\partial x^{2}}+\nu \frac{\partial^{2} W}{\partial y^{2}}\right)
$$


where $k_{0}$ and $k_{a}$ are stiffness values of the linear spring, $K_{0}$ and $K_{a}$ are stiffness values of the torsional spring, and $\nu$ is Poisson's ratio of the rectangular plate. In the process of solving equation (2.1), the solution must meet two conditions. First, the solution of the equation must be continuous and have an $n-1$ order derivative, and the $n$ order derivative must be integrable. Second, the solution must meet the boundary conditions (Eqs. (2.4) and (2.5)). In this paper, the modified Fourier-Ritz method is introduced, which is to express the solution of the equation as the Fourier series expansion and the auxiliary polynomial. In the study of beam vibration problems, this method is used to solve the problem that the traditional solution function with only the Fourier sine series expansion may cause the original function or its derivative to exhibit discontinuity at the end points (Huang and Lin, 2016). Based on this consideration, we express the displacement function of the rectangular plate as the sum of the Fourier cosine series and the auxiliary polynomial. The dynamic function is written as

$$
W(x, y)=\sum_{m=0}^{\infty}\left[A_{m} \cos \left(\lambda_{a m} x\right)+\left(p_{1}(x)+p_{2}(x)\right) \cos \left(\lambda_{b n} y\right)\right] \quad n=0,1,2, \ldots
$$

where $\lambda_{a m}=m \pi / a, \lambda_{b n}=m \pi / b, p_{1}(x)$ and $p_{2}(x)$ are the auxiliary polynomials and $A_{m}$ are unknown parameters.

The polynomial introduced must meet the following requirements

$$
\begin{aligned}
& \left(p_{1}(0)+p_{2}(0)\right) \cos \left(\lambda_{b n} y\right)=W(0, y)=\alpha_{0} \cos \left(\lambda_{b n} y\right) \\
& \left(p_{1}(a)+p_{2}(a)\right) \cos \left(\lambda_{b n} y\right)=W(a, y)=\alpha_{a} \cos \left(\lambda_{b n} y\right)
\end{aligned}
$$

and

$$
\begin{aligned}
& \frac{\partial^{2}\left(p_{1}(0)+p_{2}(0)\right)}{\partial x^{2}} \cos \left(\lambda_{b n} y\right)=\frac{\partial^{2} W(0, y)}{\partial x^{2}}=\beta_{0} \cos \left(\lambda_{b n} y\right) \\
& \frac{\partial^{2}\left(p_{1}(a)+p_{2}(a)\right)}{\partial x^{2}} \cos \left(\lambda_{b n} y\right)=\frac{\partial^{2} W(a, y)}{\partial x^{2}}=\beta_{a} \cos \left(\lambda_{b n} y\right)
\end{aligned}
$$

where $\alpha_{0}, \alpha_{a}, \beta_{0}, \beta_{a}$ are unknown parameters.

The polynomials $p_{1}(x)$ and $p_{2}(x)$ must be continuous periodic functions, so $p_{1}(x)$ can be set as the continuous lowest order polynomial

$$
p_{1}(x)=\frac{\alpha_{a} x}{a}+\frac{\alpha_{0}}{a}(a-x)
$$

and

$$
\frac{\partial^{2} p_{2}(x)}{\partial x^{2}}=\frac{\beta_{a} x}{a}+\frac{\beta_{0}}{a}(a-x)
$$

By quadratic integration of (2.10) and combining with equations (2.8), it can be concluded that

$$
p_{2}(x)=\frac{\beta_{a}}{6 a}\left(x^{3}-a^{2} x\right)-\frac{\beta_{0}}{6 a}\left(2 a^{2} x-3 a x^{2}+x^{3}\right)
$$

The Fourier sine series expansion method was discussed by Mahapatra and Panigrahi (2020). The cosine series expansion method was used to solve vibration problems of single-beam and double-beam systems, and its correctness was verified. Here, we mainly study the application of the modified Fourier-Ritz method to rectangular plates with simply supported opposite sides. 
By substituting equations (2.9), (2.11), (2.6) into equation (2.3), it can be obtained

$$
\begin{aligned}
& k_{0}\left(\alpha_{0}+\sum_{m=0}^{\infty} A_{m}\right)=-D\left(-\frac{\beta_{0}}{a}+\frac{\beta_{1}}{a}-(2-\nu) \lambda_{b n}^{2}\left(-\frac{\alpha_{0}}{a}+\frac{\alpha_{1}}{a}-\frac{a \beta_{0}}{3}-\frac{a \beta_{1}}{6}\right)\right) \\
& K_{0}\left(-\frac{\alpha_{0}}{a}+\frac{\alpha_{1}}{a}-\frac{a \beta_{0}}{3}-\frac{a \beta_{1}}{6}\right)=D\left(\beta_{0}+\sum_{m=0}^{\infty}-A_{m} \lambda_{a m}^{2}+\nu\left(-\alpha_{0} \lambda_{b n}^{2}+\sum_{m=0}^{\infty}-A_{m} \lambda_{b n}^{2}\right)\right) \\
& k_{a}\left(\alpha_{1}+\sum_{m=0}^{\infty}(-1)^{m} A_{m}\right) \\
& \quad=D\left(-\frac{\beta_{0}}{a}+\frac{\beta_{1}}{a}+(2-\nu)\left(-\lambda_{b n}^{2}\right)\left(-\frac{\alpha_{0}}{a}+\frac{\alpha_{1}}{a}+\frac{a \beta_{0}}{6}+\frac{a \beta_{1}}{3}\right)\right) \\
& K_{a}\left(-\frac{\alpha_{0}}{a}+\frac{\alpha_{1}}{a}+\frac{a \beta_{0}}{6}+\frac{a \beta_{1}}{3}\right) \\
& \quad=-D\left(\beta_{1}+\sum_{m=0}^{\infty}(-1)^{m+1} A_{m} \lambda_{a m}^{2}+\nu\left(-\alpha_{1} \lambda_{b n}^{2}+\sum_{m=0}^{\infty}(-1)^{m+1} A_{m} \lambda_{b n}^{2}\right)\right)
\end{aligned}
$$

According to (2.9) and (2.11), it can be concluded that

$$
p_{1}(x)+p_{2}(x)=\boldsymbol{\zeta}(x) \bar{\gamma}
$$

where

$$
\begin{aligned}
& \boldsymbol{\zeta}(x)=\left[\frac{a-x}{a}, \frac{x}{a}, \frac{-\left(2 a^{2} x-3 a x^{2}+x^{3}\right)}{6 a}, \frac{x^{3}-a^{2} x}{6 a}\right] \\
& \bar{\gamma}=\left[\alpha_{0}, \alpha_{a}, \beta_{0}, \beta_{a}\right]^{\mathrm{T}}
\end{aligned}
$$

According to equations (2.12), it can be found that

$$
\mathbf{H}_{n} \bar{\gamma}=\sum_{m=1}^{\infty} \mathbf{Q}_{m}^{n} A_{m}
$$

where

$$
\begin{aligned}
\mathbf{H}_{n} & =\left[\begin{array}{cccc}
-\widetilde{k}_{0}-\frac{2-\nu}{a} \lambda_{b n}^{2} & \lambda_{b n}^{2}(2-\nu) \frac{1}{a} & \frac{1}{a}-\lambda_{b n}^{2}(2-\nu) \frac{a}{3} & -\frac{1}{a}-\lambda_{b n}^{2}(2-\nu) \frac{a}{6} \\
\frac{1}{a} \widetilde{K}_{0}-\nu \lambda_{b n}^{2} & -\frac{1}{a} \widetilde{K}_{0} & \frac{a}{3} \widetilde{K}_{0}+1 & \frac{a}{6} \widetilde{K}_{0} \\
\lambda_{b n}^{2}(2-\nu) \frac{1}{a} & -\widetilde{k}_{a}-\frac{2-\nu}{a} \lambda_{b n}^{2} & -\frac{1}{a}-\lambda_{b n}^{2}(2-\nu) \frac{a}{6} & \frac{1}{a}-\lambda_{b n}^{2}(2-\nu) \frac{a}{3} \\
-\frac{1}{a} \widetilde{K}_{a} & \frac{1}{a} \widetilde{K}_{a}-\nu \lambda_{b n}^{2} & \frac{a}{6} \widetilde{K}_{a} & \frac{a}{3} \widetilde{K}_{a}+1
\end{array}\right] \\
\mathbf{Q}_{m}^{n} & =\left[\begin{array}{lll}
\widetilde{k}_{0}, \lambda_{a m}^{2}+\nu \lambda_{b n}^{2},(-1)^{m} \widetilde{K}_{a},(-1)^{m} \lambda_{a m}^{2}+\nu(-1)^{m} \lambda_{b n}^{2}
\end{array}\right]^{\mathrm{T}}
\end{aligned}
$$

where $\widetilde{k}_{0}=k_{0} / D, \widetilde{k}_{a}=k_{a} / D, \widetilde{K}_{0}=K_{0} / D, \widetilde{K}_{a}=K_{a} / D$.

By substituting Eqs, (2.13), (2.15) into Eq. (2.6), it can be obtained

$$
W(x, y)=\sum_{m=1}^{\infty} A_{m}\left[\cos \left(\lambda_{a m} x\right)+\zeta(x) \mathbf{H}_{n}^{-1} \mathbf{Q}_{m}^{n}\right] \cos \left(\lambda_{b n} y\right) \quad n=1,2, \ldots
$$


Firstly, substitute Eq. (2.17) into Eq. (2.3), and then the Galerkin discretization is adopted. Finally, the obtained equation is integrated in the whole domain of definition, which leads to

$$
\begin{aligned}
& \frac{b}{2} \lambda_{a m}^{4}\left(\frac{a}{2}+\int_{0}^{a} \cos \left(\lambda_{a m} x\right) \boldsymbol{\zeta}(x) \mathbf{H}_{\bar{n}}^{-1} \mathbf{Q}_{\bar{m}}^{\bar{n}} d x\right) \\
& \quad+2 \cdot \frac{b}{2}\left(\frac{a}{2} \lambda_{a m}^{2} \lambda_{b n}^{2}+\lambda_{a m}^{2} \lambda_{b n}^{2} \int_{0}^{a} \cos \left(\lambda_{a m} x\right) \boldsymbol{\zeta}(x) \mathbf{H}_{\bar{n}}^{-1} \mathbf{Q}_{\bar{m}}^{\bar{n}} d x\right. \\
& \left.\quad-\lambda_{b n}^{2} \int_{0}^{a} \cos \left(\lambda_{a \bar{m}} x\right)(\boldsymbol{\zeta}(x))^{\prime \prime} \mathbf{H}_{n}^{-1} \mathbf{Q}_{m}^{n} d x-\lambda_{b n}^{2} \int_{0}^{a}\left[(\boldsymbol{\zeta}(x))^{\prime \prime} \mathbf{H}_{n}^{-1} \mathbf{Q}_{m}^{n}\right]\left[\boldsymbol{\zeta}(x) \mathbf{H}_{\bar{n}}^{-1} \mathbf{Q}_{\bar{m}}^{\bar{n}}\right] d x\right) \\
& \quad+\frac{b}{2} \lambda_{b n}^{4}\left(\frac{a}{2}+\int_{0}^{a} \cos \left(\lambda_{a m} x\right) \boldsymbol{\zeta}(x) \mathbf{H}_{\bar{n}}^{-1} \mathbf{Q}_{\bar{m}}^{\bar{n}} d x+\int_{0}^{a} \cos \left(\lambda_{a \bar{m}} x\right) \boldsymbol{\zeta}(x) \mathbf{H}_{n}^{-1} \mathbf{Q}_{m}^{n} d x\right. \\
& \left.\quad+\int_{0}^{a}\left[\boldsymbol{\zeta}(x) \mathbf{H}_{n}^{-1} \mathbf{Q}_{m}^{n}\right]\left[\boldsymbol{\zeta}(x) \mathbf{H}_{\bar{n}}^{-1} \mathbf{Q}_{\bar{m}}^{\bar{n}}\right] d x\right)-\frac{\rho h \omega^{2}}{D} \frac{b}{2}\left(\frac{a}{2}+\int_{0}^{a} \cos \left(\lambda_{a m} x\right) \boldsymbol{\zeta}(x) \mathbf{H}_{\bar{n}}^{-1} \mathbf{Q}_{\bar{m}}^{\bar{n}} d x\right. \\
& \left.\quad+\int_{0}^{a} \cos \left(\lambda_{a \bar{m}} x\right) \boldsymbol{\zeta}(x) \mathbf{H}_{n}^{-1} \mathbf{Q}_{m}^{n} d x+\int_{0}^{a}\left[\boldsymbol{\zeta}(x) \mathbf{H}_{n}^{-1} \mathbf{Q}_{m}^{n}\right]\left[\boldsymbol{\zeta}(x) \mathbf{H}_{\bar{n}}^{-1} \mathbf{Q}_{\bar{m}}^{\bar{n}}\right] d x\right)=0
\end{aligned}
$$

By sorting out equation (2.18), one can obtain

$$
\left(\mathbf{K}-\frac{\rho h \omega^{2}}{D} \mathbf{M}\right) \mathbf{A}=\mathbf{0}
$$

where $(m, \bar{m}=1,2, \ldots, M, n, \bar{n}=1,2, \ldots, N)$

$$
\begin{aligned}
& \mathbf{K}_{\bar{m} \bar{n}, m n}=\delta_{\bar{n} n}\left[\left(\delta_{\bar{m} m}+\mathbf{T}_{m \bar{m}}^{\bar{n}}\right)\left(\lambda_{a m}^{2}+\lambda_{b n}^{2}\right)^{2}+\lambda_{b n}^{4}\left(\mathbf{T}_{\bar{m} m}^{n}+\mathbf{U}_{\bar{m} \bar{n}, m n}\right)-2 \lambda_{b n}^{2}\left(\widetilde{\mathbf{T}}_{\bar{m} m}^{n}+\widetilde{\mathbf{U}}_{\bar{m} \bar{n}, m n}\right)\right] \\
& \mathbf{M}_{\bar{m} \bar{n}, m n}=\delta_{\bar{n} n}\left(\delta_{\bar{m} m}+\mathbf{T}_{\bar{m} m}^{n}+\mathbf{T}_{m \bar{m}}^{\bar{n}}+\mathbf{U}_{\bar{m} \bar{n}, m n}\right) \\
& \mathbf{A}=\left[A_{11}, A_{12}, A_{13}, \ldots, A_{21}, A_{22}, A_{23}, \ldots, A_{M(N-2)}, A_{M(N-1)}, A_{M N}\right]^{\mathrm{T}} \\
& \mathbf{S}_{m n}=\mathbf{H}_{n}^{-1} \mathbf{Q}_{m}^{n} \quad \mathbf{S}_{\bar{m} \bar{n}}=\mathbf{H}_{\bar{n}}^{-1} \mathbf{Q}_{\bar{m}}^{\bar{n}} \\
& \mathbf{T}_{\bar{m} m}^{n}=\mathbf{P}_{\bar{m}} \mathbf{S}_{m n} \quad \widetilde{\mathbf{T}}_{\bar{m} m}^{n}=\widetilde{\mathbf{P}}_{\bar{m}} \mathbf{S}_{m n} \\
& \mathbf{U}_{m n, \bar{m} \bar{n}}=\left(\mathbf{S}_{m n}\right)^{\mathrm{T}} \mathbf{Z} \mathbf{S}_{\bar{m} \bar{n}} \quad \widetilde{\mathbf{U}}_{m n, \bar{m} \bar{n}}=\left(\mathbf{S}_{m n}\right)^{\mathrm{T}} \widetilde{\mathbf{Z}} \mathbf{S}_{\bar{m} \bar{n}} \\
& \mathbf{P}_{m}=\frac{2}{a} \int_{0}^{a} \boldsymbol{\zeta}(x) \cos \left(\lambda_{a m} x\right) d x \\
& =\frac{2}{a}\left[\frac{1-(-1)^{m}}{a \lambda_{a m}^{2}}, \frac{-1+(-1)^{m}}{a \lambda_{a m}^{2}}, \frac{\mathcal{P}_{1}}{6 a \lambda_{a m}^{4}}, \frac{\mathcal{P}_{2}}{6 a \lambda_{a m}^{4}}\right] \\
& \mathcal{P}_{1}=-6+2 a \lambda_{a m}^{2}+(-1)^{m}\left(6+a^{2} \lambda_{a m}^{2} \quad \mathcal{P}_{2}=6+a^{2} \lambda_{a m}^{2}+2(-1)^{m}\left(-3+a^{2} \lambda_{a m}^{2}\right)\right. \\
& \widetilde{\mathbf{P}}_{m}=\frac{2}{a} \int_{0}^{a}(\boldsymbol{\zeta}(x))^{\prime \prime} \cos \left(\lambda_{a m} x\right) d x=\frac{2}{a}\left[0,0, \frac{1-(-1)^{m}}{a \lambda_{a m}^{2}}, \frac{-1+(-1)^{m}}{a \lambda_{a m}^{2}}\right] \\
& \mathbf{Z}=\frac{2}{a} \int_{0}^{a}(\boldsymbol{\zeta}(x))^{\mathrm{T}} \boldsymbol{\zeta}(x) d x=\frac{2}{a}\left[\begin{array}{cccc}
\frac{a}{3} & \frac{a}{6} & -\frac{a^{3}}{45} & -\frac{7 a^{3}}{360} \\
\frac{a}{6} & \frac{a}{3} & -\frac{7 a^{3}}{360} & -\frac{a^{3}}{45} \\
-\frac{a^{3}}{45} & -\frac{7 a^{3}}{360} & \frac{2 a^{5}}{945} & \frac{31 a^{3}}{15120} \\
-\frac{7 a^{3}}{360} & -\frac{a^{3}}{45} & \frac{31 a^{3}}{15120} & \frac{2 a^{5}}{945}
\end{array}\right]
\end{aligned}
$$




$$
\widetilde{\mathbf{Z}}=\frac{2}{a} \int_{0}^{a}(\boldsymbol{\zeta}(x))^{\mathrm{T}}(\boldsymbol{\zeta}(x))^{\prime \prime} d x=\frac{2}{a}\left[\begin{array}{cccc}
0 & 0 & \frac{a}{3} & \frac{a}{6} \\
0 & 0 & \frac{a}{6} & \frac{a}{3} \\
0 & 0 & -\frac{a^{3}}{45} & -\frac{7 a^{3}}{360} \\
0 & 0 & -\frac{7 a^{3}}{360} & -\frac{a^{3}}{45}
\end{array}\right]
$$

By solving the matrix eigenvalue problem of equation (2.19), we can easily obtain the natural frequency and the corresponding modal shape of the rectangular plate. By changing the stiffness values of linear and torsional springs, different boundary conditions can be solved (in the solution process, the values of $m$ and $n$ were truncated to $M$ and $N$, respectively).

\section{Numerical examples and discussions}

In this Section, the accuracy and convergence of the above method will be demonstrated and compared with the traditional method and results of some researchers. At the same time, the vibration problem of a rectangular plate with elastic constraints along the edge will be discussed by changing the stiffness value of the spring and the aspect ratio of the rectangular plate.

\subsection{Convergence and accuracy analysis}

In order to verify the accuracy and convergence of this method, firstly, we calculate the vibration frequency of a square plate simply supported and fixed along the opposite sides by setting the stiffness of four springs to be infinite $\left(10^{10}\right.$ here, which will be discussed in the next Section). The traditional use of a single sine function to solve the equation is (Cao, 1989)

$$
\left(\beta_{1 m}^{2}-\beta_{2 m}^{2}\right) \sin \left(\beta_{1 m} b\right) \sinh \left(\beta_{2 m} b\right)-2 \beta_{1 m} \beta_{2 m}\left[\cos \left(\beta_{1 m} b\right) \cosh \left(\beta_{2 m} b\right)-1\right]=0
$$

where

$$
\beta_{1 m}=\sqrt{a^{2}-\left(\frac{m \pi}{a}\right)^{2}} \quad \beta_{2 m}=\sqrt{a^{2}+\left(\frac{m \pi}{a}\right)^{2}}
$$

In the process of calculation, we generally perform dimensionless processing on the calculated frequency to display the calculation result, that is, use $\varpi=\omega a^{2}(\rho h / D)^{1 / 2}$ to display the data. Table 1 shows the results of calculating the lowest sixth-order frequency parameter $\varpi$ with different truncation coefficients $M(N=10)$. It also includes the data calculated by Eq. (3.1) and the data studied by the researcher. In addition, we also use the finite element software for calculation, in which the density of the rectangular thin plate is $\rho=7850 \mathrm{~kg} / \mathrm{m}^{3}$, elastic modulus is $E=2.0 \cdot 10^{11} \mathrm{~Pa}$, length $a=2 \mathrm{~m}$, and Poisson's ratio $\nu=0.3$. The finite element method is modeled by Shell181 element in Workbench. The division element size is $0.03 \mathrm{~m}$ and the grid type adopts quadrilateral grid division formula (the same parameters are used below). Through comparison, it can be seen that this method has faster convergence and higher accuracy.

Next, we study vibration of a typical rectangular plate with a free boundary. By setting the parameters $\widetilde{k}_{0}=10^{10}$ and $\widetilde{k}_{a}=\widetilde{K}_{0}=\widetilde{K}_{a}=0$ of four springs, respectively, the simple support along the $x=0$ direction and the free edge along the $x=a$ direction is realized. Since vibration of a rectangular plate with a free edge is related to Poisson's ratio $\nu, \nu=0.3$ in the calculation process, and the corresponding traditional frequency parameter calculation formula is (Cao, 1989)

$$
\lambda_{2}\left[\Omega-(1-\nu) n^{2} \pi^{2}\right]^{2} \sin \lambda_{1} \cosh \lambda_{2}-\lambda_{1}\left[\Omega+(1-\nu) n^{2} \pi^{2}\right]^{2} \cos \lambda_{1} \sinh \lambda_{2}=0
$$

where

$$
\Omega=b^{2} \omega \sqrt{\frac{\rho h}{D}} \quad \lambda_{1,2}=\frac{a}{b} \sqrt{\Omega \mp n^{2} \pi^{2}}
$$


Table 1. Frequency parameters of the C-S-C-S plate with the increasing truncation coefficient $M$

\begin{tabular}{|c|c|c|c|c|c|c|}
\hline \multirow{2}{*}{$M$} & \multicolumn{6}{|c|}{ Frequency parameter $\varpi$ order } \\
\cline { 2 - 7 } & 1st & 2nd & 3rd & 4th & 5 th & 6 th \\
\hline \hline 6 & 29.0131 & 54.8626 & 69.327 & 94.5863 & 102.405 & 130.376 \\
\hline 8 & 28.9808 & 54.8018 & 69.327 & 94.5855 & 102.312 & 129.706 \\
\hline 10 & 28.9674 & 54.776 & 69.327 & 94.5853 & 102.271 & 129.433 \\
\hline 12 & 28.961 & 54.7633 & 69.327 & 94.5853 & 102.250 & 129.301 \\
\hline 20 & 28.9533 & 54.748 & 69.327 & 94.5853 & 102.225 & 129.145 \\
\hline Eq. (3.1) & 28.9444 & 54.7452 & 69.3223 & 94.5756 & 102.2121 & 129.0496 \\
\hline Zhang et al. (2020) & 28.9509 & 54.7431 & 69.3270 & 94.5853 & 102.2160 & 129.096 \\
\hline Workbench & 28.9620 & 54.7819 & 69.4259 & 94.6927 & 102.4160 & 129.49 \\
\hline
\end{tabular}

Table 2 shows the results of calculating the lowest sixth-order frequency parameter $\varpi$ when taking different truncation coefficients $M(N=10)$. It also includes the data calculated by Eq. (3.3), the data studied by the researcher and the results of Workbench. The comparison shows that the method of Fourier cosine series expansion is consistent with the results calculated by traditional methods.

Table 2. Frequency parameters of the S-S-F-S plate with the increasing truncation coefficient $M$

\begin{tabular}{|c|c|c|c|c|c|c|}
\hline \multirow{2}{*}{$M$} & \multicolumn{6}{|c|}{ Frequency parameter $\varpi$ order } \\
\cline { 2 - 7 } & 1st & 2nd & 3rd & 4th & 5th & 6th \\
\hline \hline 6 & 11.6907 & 27.7569 & 41.2583 & 59.1643 & 62.3863 & 90.5934 \\
\hline 8 & 11.6872 & 27.7566 & 41.223 & 59.1077 & 61.0816 & 90.0027 \\
\hline 10 & 11.6859 & 27.7565 & 41.2102 & 59.0872 & 61.9743 & 90.3605 \\
\hline 12 & 11.6854 & 27.7564 & 41.2045 & 59.0781 & 61.9269 & 90.3323 \\
\hline 20 & 11.6847 & 27.7564 & 41.1984 & 59.0683 & 61.8752 & 90.3023 \\
\hline Eq. (3.3) & 11.6845 & 27.7564 & 41.1967 & 59.0659 & 61.8606 & 90.2948 \\
\hline$[20]$ & 11.7609 & 27.8126 & 41.6851 & 59.5089 & - & - \\
\hline Workbench & 11.6853 & 27.7569 & 41.2370 & 59.0994 & 61.9098 & 90.5002 \\
\hline
\end{tabular}

[20] - Mahapatra and Panigrahi (2020)

Through the comparison of the data of the two sets of different boundary conditions in Tables 1 and 2, it can be found that the results calculated by Eq. (2.19) are consistent with the results obtained by traditional research. There are free edges in the boundary conditions in Table 2, which is more illustrative for verification of the results. At the same time, Tables 1 and 2 show that with an increase in the truncation coefficient $M$, the results tend to be more stable, which verifies the convergence of the Fourier cosine series expansion.

\subsection{Boundary condition analysis}

This paper studies vibration of a rectangular plate with elastic constraints along the edges. One can solve different constraints by setting the stiffness of the elastic constraint, for example, by setting the stiffness of two linear springs to be infinite and stiffness of the two torsional springs to be infinitesimal in order to realize the simply supported constraint of the rectangular plate, but now there is no way to determine whether the data substituted in the calculation process is infinitely large or infinitely small. Therefore, the influence of spring stiffness of different sizes on the frequency parameter $\varpi=\omega a^{2}(\rho h / D)^{1 / 2}$ needs to be discussed in detail. In the calculation process, take the truncation coefficient $M=N=10$, the stiffness of one spring is changed 
respectively. As shown in Fig. 2, the results of the lowest fourth-order frequency parameter $\varpi$ are changing with different stiffness values of different types of springs.

(a)

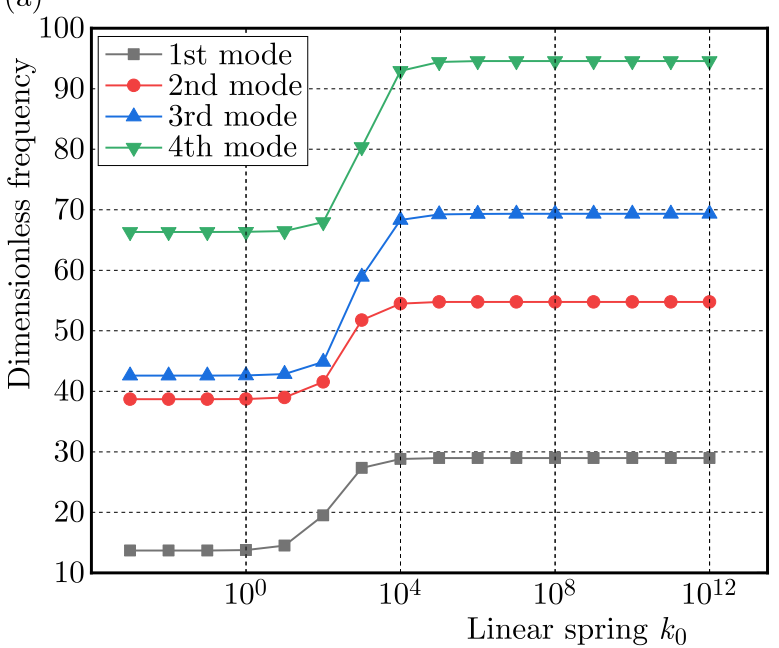

(c)

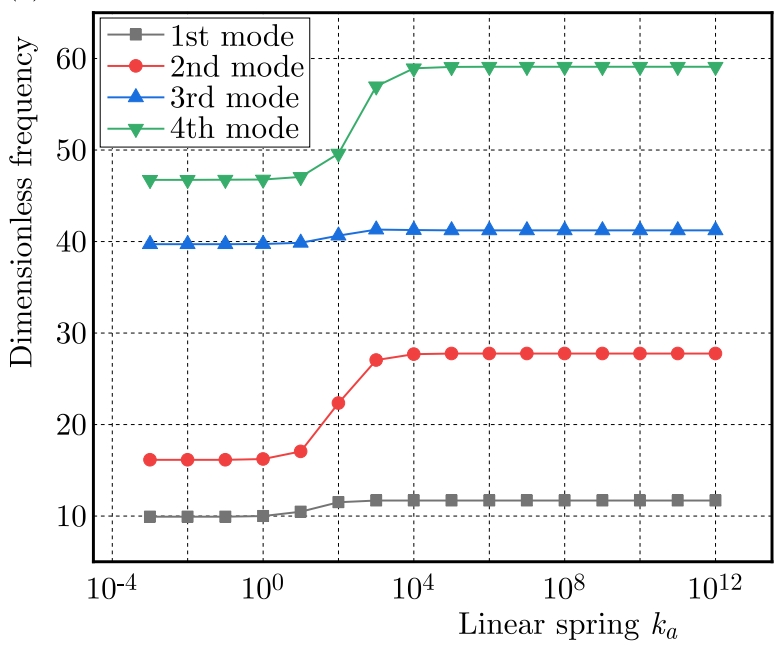

(b)

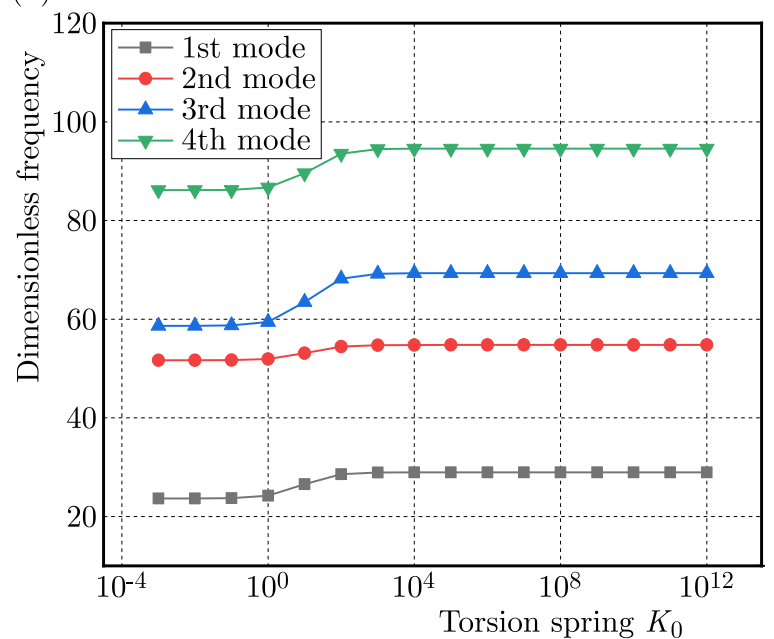

(d)

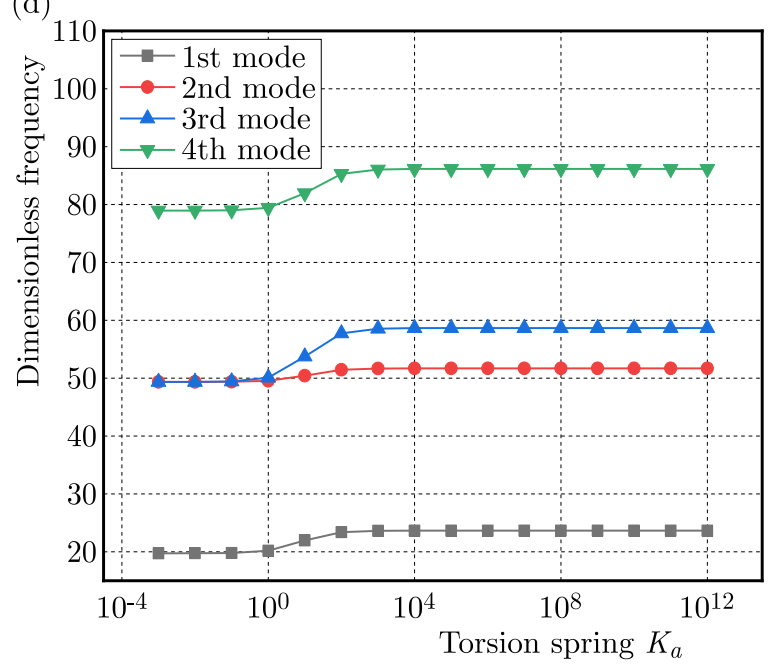

Fig. 2. Values of the dimensionless parameter $\varpi$ corresponding to different spring stiffness

In Fig. 2, the variation of frequency parameters under different boundary constraints is basically the same. As the spring stiffness value increases, the boundary conditions slowly evolve into the well-known simple support constraint. When the stiffness value exceeds $10^{4}$, the frequency parameters basically tend to be stable, that is, the set constraint conditions are reached. For the accuracy of calculation, this paper takes the infinity as $10^{10}$ and the infinitesimally small value as $10^{-2}$ (to avoid calculation of singular matrices).

\subsection{Analysis of other typical boundary conditions}

Tables 1 and 2 respectively calculated the frequency parameters of rectangular plates with simply supported boundaries and free boundaries, which proved the accuracy and convergence of the modified Fourier-Ritz method. Now we consider vibration of a rectangular plate constrained by an elastic support along the $x=0, a$ direction. We set the linear spring stiffness to be $\widetilde{k}_{0}=\widetilde{k}_{a}=10^{10}$, while the torsional spring changes from $\widetilde{K}_{0}=\widetilde{K}_{a}=0$ to $\widetilde{K}_{0}=\widetilde{K}_{a}=\infty$. In this way, the boundary conditions evolve into typical simply supported and fixed supported cases. In the calculation process, the truncation coefficient $M=20$ and the frequency parameter $\varpi_{1}=\omega^{2} a^{4} \rho h /\left(\pi^{4} D\right)$ are taken. The calculation results are shown in Table 3. 
Table 3. Frequency parameters of the S-S-S-S plate with the increasing $\widetilde{K}_{0}$ and $\widetilde{K}_{a}$

\begin{tabular}{|c|c|c|c|c|c|c|}
\hline \multirow{2}{*}{$\widetilde{K}_{0}=\widetilde{K}_{a}$} & \multicolumn{6}{|c|}{ Frequency parameter $\varpi_{1}$ order } \\
\cline { 2 - 7 } & 1st & 2nd & 3rd & 4th & 5 th & 6 th \\
\hline \hline 0 & 4.00012 & 25.0 & 100.087 & 289.0 & 677.908 & 1369.0 \\
\hline Li and Daniels (2002) & 4.0 & 25.0 & 100.0 & 289.0 & - & - \\
\hline 10 & 6.1698 & 34.8307 & 124.753 & 335.975 & 756.074 & 1484.59 \\
\hline Li and Daniels (2002) & 6.16826 & 34.8307 & 124.569 & 335.975 & - & - \\
\hline 100 & 8.14601 & 46.2672 & 160.343 & 417.339 & 911.333 & 1747.12 \\
\hline Li and Daniels (2002) & 8.14370 & 46.2673 & 160.131 & 417.339 & - & - \\
\hline 1000 & 8.55552 & 48.9957 & 169.974 & 442.468 & 964.124 & 1848.06 \\
\hline$\vdots$ & $\vdots$ & $\vdots$ & $\vdots$ & $\vdots$ & $\vdots$ & $\vdots$ \\
\hline$\infty$ & 8.60589 & 49.3407 & 171.22 & 445.822 & 971.306 & 1862.28 \\
\hline Li and Daniels (2002) & 8.60448 & 49.3408 & 171.089 & 445.823 & - & - \\
\hline
\end{tabular}

(a)

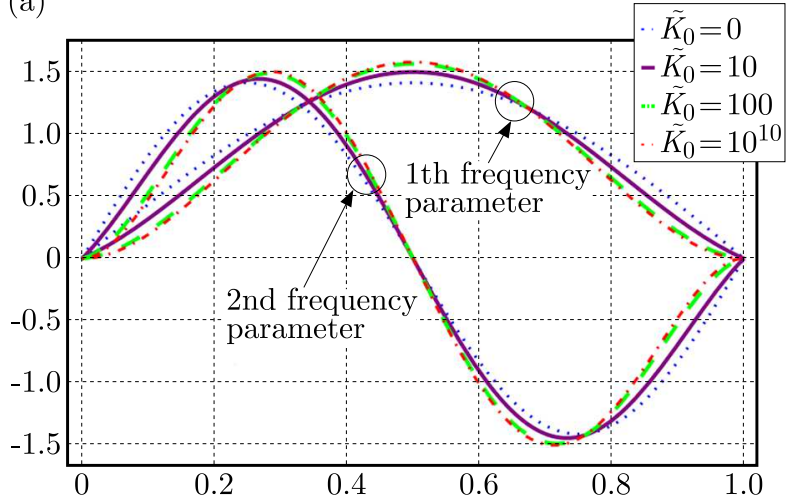

(c)

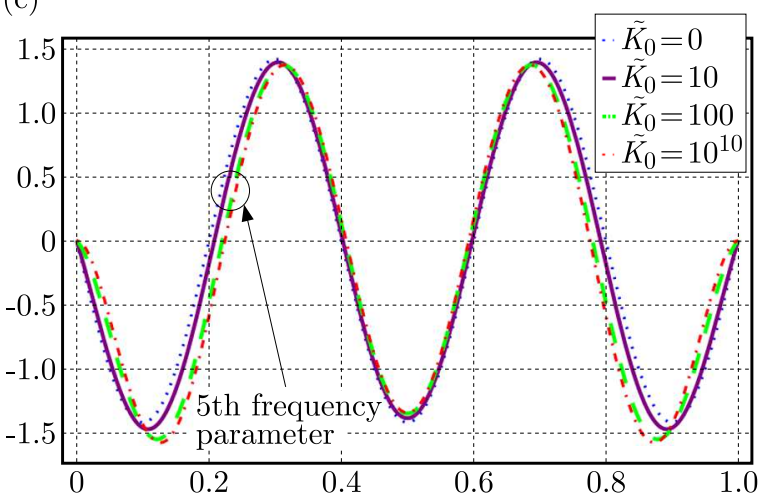

(b)

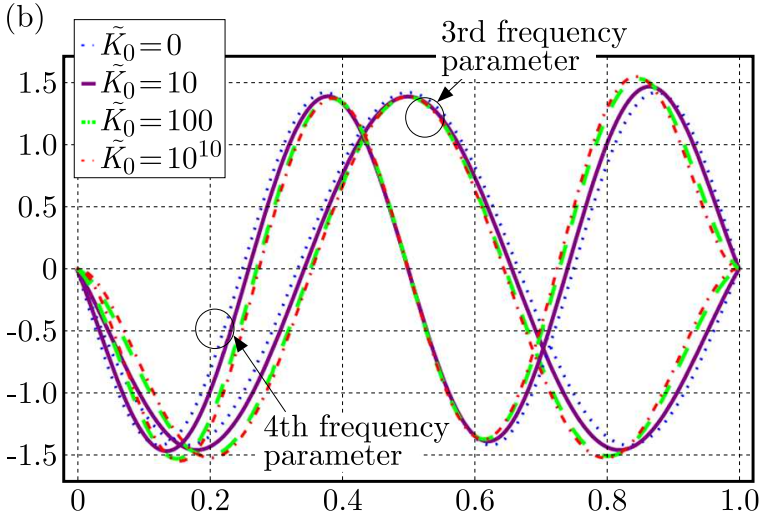

(d)

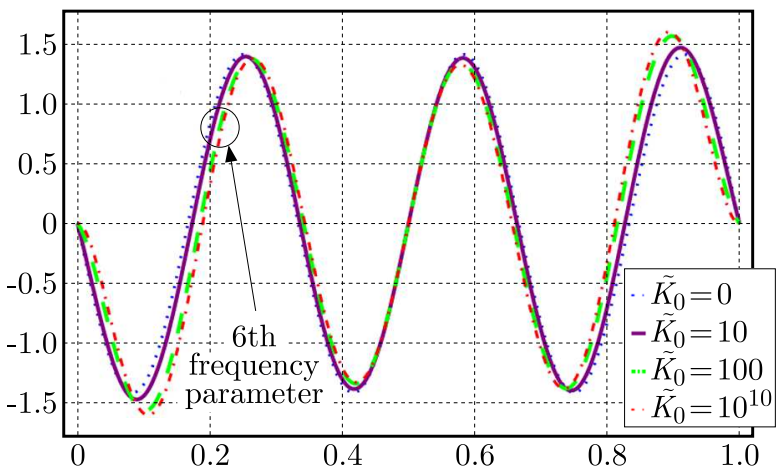

Fig. 3. Mode shapes corresponding to the lowest sixth-order frequency parameter $\varpi=\omega a^{2}(\rho h / D)^{1 / 2}$ at $y=b / 2$

The data in Table 3 shows that the modified Fourier-Ritz method is also consistent with the results of other researchers in the rectangular plate with the torsional constraint. In order to better understand the effect of stiffness of the torsional spring on the mode shape, the vibration mode corresponding to the lowest sixth order frequency parameter $\varpi=\omega a^{2}(\rho h / D)^{1 / 2}$ at $y=b / 2$ (truncation coefficient $N=1$ ) is shown in Fig. 3. It can be seen from the figure that with an increase in the spring stiffness, the amplitude slightly increases, and the vibration mode towards the boundary becomes more and more flat, which is in line with our common sense. 
In the previous calculation, square plates are taken as examples. Here, we consider the change of the frequency parameter $\varpi_{1}=\omega^{2} a^{2} b^{2} \rho h /\left(\pi^{4} D\right)$ of a rectangular plate with different aspect ratios as the torsional spring stiffness increases, and take the truncation coefficient $M=10$. The first-order frequency parameters are shown in Table 4. It can be seen from the table that the method described in this paper still has good accuracy, and we can also know that the frequency parameters increase regularly with the increase of torsional spring stiffness under the same aspect ratio.

Table 4. Frequency parameters of the S-S-S-S plate with different aspect ratios as $\widetilde{K}_{0}$ and $\widetilde{K}_{a}$ increase

\begin{tabular}{|c|c|c|c|c|c|c|}
\hline \multirow{2}{*}{$\widetilde{K}_{0}=\widetilde{K}_{a}$} & \multicolumn{6}{|c|}{ Frequency parameter $\varpi_{1}$ order } \\
\cline { 2 - 7 } & \multicolumn{2}{|c|}{$b / a=0.5$} & \multicolumn{2}{c|}{$b / a=1.0$} & \multicolumn{2}{c|}{$b / a=1.5$} \\
\cline { 2 - 7 } & Present & Cao (1989) & Present & Cao $(1989)$ & Present & Cao (1989) \\
\hline \hline 0 & 6.25003 & 6.25 & 4.00012 & 4.0 & 4.6947 & 4.694 \\
\hline 1 & 6.34486 & 6.333 & 4.37341 & 4.373 & 5.5308 & 5.530 \\
\hline 10 & 6.84843 & 6.847 & 6.1698 & 6.168 & 9.44909 & 9.446 \\
\hline 100 & 7.51621 & 7.514 & 8.14601 & 8.144 & 13.5607 & 13.557 \\
\hline 1000 & 7.67292 & - & 8.55552 & - & 14.3879 & - \\
\hline$\infty$ & 7.69267 & 7.691 & 8.60589 & 8.604 & 14.4891 & 14.487 \\
\hline
\end{tabular}

In addition, according to the solution to equation $(2.12)_{3}$, when the boundary condition contains the free boundary, the frequency parameter is related to Poisson's ratio $\nu$. By setting $\widetilde{k}_{0}=10^{10}$ and $\widetilde{k}_{a}=\widetilde{K}_{0}=\widetilde{K}_{a}=0$, we discuss the simple support along the $x=0$ direction and the free edge along the $x=a$ direction. Take the frequency parameter $\varpi=\left[\omega b^{2}(\rho h / D)^{1 / 2}\right]^{1 / 2}$ and the truncation coefficient $M=20$. The first-order frequency parameters with different aspect ratios and Poisson's ratio are shown in Table 5. The data in Table 5 shows the accuracy of the modified Fourier-Ritz method. It is also found that different Poisson's ratios have more complicated effects on rectangular plates with free edges.

Table 5. Frequency parameters of the S-S-F-S plate with different aspect ratios and Poisson's ratio

\begin{tabular}{|c|c|c|c|c|c|c|}
\hline \multirow{2}{*}{$b / a$} & \multicolumn{6}{|c|}{ Frequency parameter $\varpi$ order } \\
\cline { 2 - 7 } & \multicolumn{2}{|c|}{$\nu=0.3$} & \multicolumn{2}{c|}{$\nu=1 / 3$} & \multicolumn{2}{c|}{$\nu=0.5$} \\
\cline { 2 - 7 } & Present & Workbench & Present & Cao (1989) & Present & Cao (1989) \\
\hline \hline 0.4 & 3.1822 & 3.1845 & 3.1764 & 3.176 & 3.1262 & 3.126 \\
\hline $2 / 3$ & 3.2667 & 3.2674 & 3.2571 & 3.257 & 3.1827 & 3.183 \\
\hline 1.0 & 3.4183 & 3.3539 & 3.4032 & 3.403 & 3.2969 & 3.297 \\
\hline 1.5 & 3.7029 & 3.7027 & 3.6791 & 3.679 & 3.5255 & 3.525 \\
\hline 2.5 & 4.336 & 4.3357 & 4.297 & 4.298 & 4.0626 & 4.063 \\
\hline
\end{tabular}

Finally, consider a more complicated boundary condition. The square plate is clamped along the $x=0$ direction, and there are elastic and linear constraints along the $x=a$ direction. We set the boundary conditions as $\widetilde{k}_{0}=\widetilde{K}_{0}=10^{10}, \widetilde{k}_{a}=100$ and $\widetilde{K}_{a}=10$ to calculate this case, where the frequency parameter is $\varpi=\omega a^{2}(\rho h / D)^{1 / 2}$. Table 6 shows the results of the first six-order frequency parameters and different truncation coefficient combinations calculated by this method. The results calculated by the previous researchers are attached. The data shows that this method has good convergence and accuracy. 
Table 6. Frequency parameters of the S-S-F-S plate with the increasing truncation coefficient $M$

\begin{tabular}{|c|c|c|c|c|c|c|}
\hline \multirow{2}{*}{$M$} & \multicolumn{7}{|c|}{ Frequency parameter $\varpi$ order } \\
\cline { 2 - 7 } & 1st & 2nd & 3rd & 4th & 5th & 6 th \\
\hline \hline 6 & 19.4188 & 40.7813 & 44.8837 & 67.2078 & 81.564 & 92.7143 \\
\hline 8 & 19.3991 & 40.7715 & 44.8566 & 67.1878 & 81.317 & 92.6633 \\
\hline 10 & 19.3916 & 40.7676 & 44.8462 & 67.1788 & 81.2237 & 92.6441 \\
\hline 12 & 19.3881 & 40.7657 & 44.8415 & 67.1743 & 81.1808 & 92.6352 \\
\hline 20 & 19.3843 & 40.7636 & 44.8361 & 67.1689 & 81.1326 & 92.6253 \\
\hline Leissa (1993) & 19.3982 & 40.7187 & 44.8104 & 67.0422 & 81.0514 & 92.5214 \\
\hline
\end{tabular}

\section{Conclusions}

In this paper, the modified Fourier-Ritz method for the analysis and calculation of rectangular plates vibration is introduced. The method is mainly applied to a rectangular plate with a set of simply supported opposite sides and another set of arbitrary elastic constraints. Differently from the classical method, in order to the displacement function of the rectangular plate in the traditional method and its derivative to be discontinuous or non-derivable at the boundary, the displacement function is expressed in the form of the sum of standard cosine series and periodic polynomial functions, by solving the eigenvalues of the matrix to obtain the mode of the rectangular plate. In order to verify the accuracy and convergence of the modified Fourier-Ritz method, several numerical examples are given in this paper. Compared with the results of the traditional calculation method, the data shows that the method has the advantages of fast convergence and high accuracy. Moreover, the boundary conditions are also analyzed in the article, and different spring stiffness settings can evolve into different boundary conditions. The method studied in this paper is helpful to the design of rectangular plate structures and the study of vibration control.

\section{References}

1. Abdulkerim S., Dafnis A., Riemerdes H., 2019, Experimental investigation of nonlinear vibration of a thin rectangular plate, International Journal of Applied Mechanics, 11, 267-289

2. Alkhayal J., Chehab J.P., Jazar M., 2019, Existence, uniqueness, and numerical simulations of Föppl-von Kármán equations for simply supported plate, Mathematical Methods in the Applied Sciences, 42, 7482-7493

3. Bahrami A., Bahrami M.N., Ilkhani M.R., 2014, Comments on "New exact solutions for free vibrations of thin orthotropic rectangular plates", Composite Structures, 107, 745-746

4. Banerjee J.R., Papkov S.O., Liu X., Kennedy D., 2015, Dynamic stiffness matrix of a rectangular plate for the general case, Journal of Sound and Vibration, 342, 177-199

5. Bert C.W., Devarakonda K.K., 2003, Buckling of rectangular plates subjected to nonlinearly distributed in-plane loading, International Journal of Solids and Structures, 40, 4097-4106

6. CAo Z.Y., 1989, Vibration Theory of Plates and Shells, China Railway Publishing House, Beijing

7. Du J., Li W.L., Jin G., Yang T., Liu Z., 2007, An analytical method for the in-plane vibration analysis of rectangular plates with elastically restrained edges, Journal of Sound and Vibration, 306, 908-927

8. Eisenberger M., Deutsch A., 2019, Solution of thin rectangular plate vibrations for all combinations of boundary conditions, Journal of Sound and Vibration, 452, 1-12

9. Gavalas G.R., El-Raheb M., 2014, Extension of Rayleigh-Ritz method for eigenvalue problems with discontinuous boundary conditions applied to vibration of rectangular plates, Journal of Sound and Vibration, 333, 4007-4016 
10. Han Qingkai Z.J.Z.H., 2016, Fundamental and Numerical Simulation on Machinery Dynamics and Vibration, Wuhan University of Technology Press

11. Hao Q., Zhai W., Chen Z., 2018, Free vibration of connected double-beam system with general boundary conditions by a modified Fourier-Ritz method, Archive of Applied Mechanics, 88, 741-754

12. He W., Chen W., Qiao H., 2013, In-plane vibration of rectangular plates with periodic inhomogeneity: Natural frequencies and their adjustment, Composite Structures, 105, 134-140

13. HuANG C.S., Lin Y.J., 2016, Fourier series solutions for vibrations of a rectangular plate with a straight through crack, Applied Mathematical Modelling, 40, 10389-10403

14. Ilanko S., Monterrubio L.E., Mochida Y., 2014, The Rayleigh-Ritz Method for Structural Analysis, Wiley, Somerset

15. Leissa A.W., 1993, Vibration of Plates, Acoustical Society of America, Washington, D.C

16. Li R., Wang H., Zheng X., Xiong S., Hu Z., Yan X., Xiao Z., Xu H., Li P., 2019, New analytic buckling solutions of rectangular thin plates with two free adjacent edges by the symplectic superposition method, European Journal of Mechanics - A/Solids, 76, 247-262

17. Li W.L., 2001, Dynamic analysis of beams with arbitrary elastic supports at both ends, Journal of Sound and Vibration, 246, 751-756

18. Li W.L., DAniels M., 2002, A Fourier series method for the vibrations of elastically restrained plates arbitrarily loaded with springs and masses, Journal of Sound and Vibration, 252, 768-781

19. LiU X., BANERJEe J.R., 2016, Free vibration analysis for plates with arbitrary boundary conditions using a novel spectral-dynamic stiffness method, Computers and Structures, 164, 108-126

20. Mahapatra K., Panigrahi S.K., 2020, Dynamic response and vibration power flow analysis of rectangular isotropic plate using Fourier series approximation and mobility approach, Journal of Vibration Engineering and Technologies, 8, 105-119

21. Najarzadeh L., Movahedian B., Azhari M., 2018, Free vibration and buckling analysis of thin plates subjected to high gradients stresses using the combination of finite strip and boundary element methods, Thin-Walled Structures, 123, 36-47

22. Shi D., Wang Q., Shi X., PAng F., 2015, An accurate solution method for the vibration analysis of Timoshenko beams with general elastic supports, Proceedings of the Institution of Mechanical Engineers. Part C, Journal of Mechanical Engineering Science, 229, 2327-2340

23. Shi X., Shi D., Li W.L., WAng Q., 2016, A unified method for free vibration analysis of circular, annular and sector plates with arbitrary boundary conditions, Journal of Vibration and Control, 22, 442-456

24. WANG X., GAN L., WANG Y., 2006, A differential quadrature analysis of vibration and buckling of an SS-C-SS-C rectangular plate loaded by linearly varying in-plane stresses, Journal of Sound and Vibration, 298, 420-431

25. Wei Z., Yin X., Yu S., Wu W., 2020, Dynamic stiffness formulation for transverse and inplane vibration of rectangular plates with arbitrary boundary conditions based on a generalized superposition method, International Journal of Mechanics and Materials in Design, 17, 1-17

26. Xing Y.F., Xu T.F., 2013, Solution methods of exact solutions for free vibration of rectangular orthotropic thin plates with classical boundary conditions, Composite Structures, 104, 187-195

27. Zhang J., Lu J., Ullah S., Gao Y., Zhao D., 2020, Buckling analysis of rectangular thin plates with two opposite edges free and others rotationally restrained by finite Fourier integral transform method, ZAMM - Journal of Applied Mathematics and Mechanics/Zeitschrift für Angewandte Mathematik und Mechanik, 101, 4

28. Zhang J., Ullah S., ZhONG Y., 2020, Accurate free vibration solutions of orthotropic rectangular thin plates by straightforward finite integral transform method, Archive of Applied Mechanics, 90, $353-368$ 